\title{
Polyhedral Methods in Numerical Algebraic Geometry*
}

\author{
Jan Verschelde ${ }^{\dagger}$ \\ to Andrew Sommese, on his $60^{\text {th }}$ birthday
}

\begin{abstract}
In numerical algebraic geometry witness sets are numerical representations of positive dimensional solution sets of polynomial systems. Considering the asymptotics of witness sets we propose certificates for algebraic curves. These certificates are the leading terms of a Puiseux series expansion of the curve starting at infinity. The vector of powers of the first term in the series is a tropism. For proper algebraic curves, we relate the computation of tropisms to the calculation of mixed volumes. With this relationship, the computation of tropisms and Puiseux series expansions could be used as a preprocessing stage prior to a more expensive witness set computation. Systems with few monomials have fewer isolated solutions and fewer data are needed to represent their positive dimensional solution sets.
\end{abstract}

2000 Mathematics Subject Classification. Primary 65H10. Secondary 14Q99, 68W30.

Key words and phrases. certificate, mixed volume, Newton polytope, numerical algebraic geometry, polyhedral method, polynomial system, Puiseux series, tropism, witness set.

\section{Introduction}

Solving polynomial systems numerically used to be restricted to finding approximations to all isolated solutions. Via numerical algebraic geometry $[69,70]$ we are now able to provide numerical representations for all solutions and for all dimensions. The development of the methods of numerical algebraic geometry coincided with upgrades [67] to the software PHCpack [76]. Its blackbox solver computes isolated solutions via polyhedral methods. The aim of this paper is to bring polyhedral methods to the foreground and to show how they may lead to finer representations of solution sets.

We consider as given a polynomial system with at least as many equations as unknowns, and we want to determine whether a proper algebraic curve is a solution. An algebraic curve is proper if it is not contained in any higher dimensional solution set. Moreover, we will assume that the proper algebraic curves we are looking for are of multiplicity one. What we want to determine of a solution curve is a certificate of its existence. The certificate should be small and straightforward to use in computer algebra systems.

*Date: 31 December 2008. This material is based upon work supported by the National Science Foundation under Grant No. 0713018.

${ }^{\dagger}$ Department of Mathematics, Statistics, and Computer Science, University of Illinois at Chicago, 851 South Morgan (M/C 249), Chicago, IL 60607-7045, USA. Email: jan@math.uic.edu or jan.verschelde@na-net.ornl.gov. URL: http://www.math.uic.edu/ jan. 
For the solution to this problem we propose polyhedral methods to find the leading terms of a Puiseux series expansion [60] of the solution curve. Verifying the existence of a curve as a solution of the given polynomial system requires then only the formal substitution of the Puiseux series into the given system, a routine task for computer algebra systems. The main source of inspiration for this approach was found in the emerging field of tropical algebraic geometry [36], [61], [73]. To prove that a polynomial system from celestial mechanics has only finitely many isolated solutions, polyhedral methods were applied in [33]. This paper could be seen as an attempt to make such proofs automatic.

Working with series expansions is a hybrid form of computation, combining symbolic and numerical approaches. Some algebraic curves may actually have finite series expansions and in the extreme case even consist of only one leading term. When considering systems with natural parameters, such solution curves may be more useful than other solutions and it may be worthwhile to look for such solutions first. Our goal is to develop a polyhedral method that will focus on computing one dimensional solutions.

After defining witness sets and outlining our problem statement, we will introduce tropisms for binomial systems. Proper algebraic curves defined by binomial systems admit a very explicit solution. To examine the normalization of tropisms we consider deformations of witness sets. The link between tropisms and mixed volumes passes through the second theorem of Bernshtein [8]. We show how to compute tropisms to proper algebraic curves defined by square systems via a special lifting.

In the spirit of the theme of the conference, held in honor of Andrew Sommese, 22-24 May 2008 , this paper is on the interactions of classical algebraic geometry — in particular the Puiseux series, the roots of polyhedral methods and tropical algebraic geometry - and the new field of numerical algebraic geometry. Detailed descriptions of algorithms and their implementations are still a work in progress.

Acknowledgements. Preliminary versions of the ideas in this paper were presented at four conferences held respectively in Tobago, Notre Dame, Hong Kong, and Vancouver. The author thanks all organizers of these meetings for their invitations and the participants for their feedback. The paper benefited from remarks suggested by a referee.

\section{Witness Sets and Newton Polytopes}

In numerical algebraic geometry, algbraic sets are manipulated by homotopy continuation methods [46], [54], [70]. The key data representation for algebraic sets is a witness set (first defined as witness point set in [66], see also [70]), defined below.

Definition 2.1 Given a system $\mathbf{f}(\mathbf{x})=\mathbf{0}$, we represent a component of $\mathbf{f}^{-1}(\mathbf{0})$ of dimension $k$ in $\mathbb{C}^{n}$ and degree $d$ by a witness set which consists of $\mathbf{f}$, the intersection $L$ of $k$ general hyperplanes (so $\operatorname{dim}(L)=n-k$ ), and $d$ generic points in $\mathbf{f}^{-1}(\mathbf{0}) \cap L$.

A generic choice of coefficients for the $k$ hyperplanes implies that all solutions will be isolated and regular, unless the component has a multiplicity higher than one. Definition 2.1 incorporates the theorem of Bertini, see [70] for its application in numerical algebraic geometry. Bertini [6] is 
a more recent software system for numerical algebraic geometry. A dictionary compares in [68] witness sets to lifting fibers [28], [30] in a geometric resolution [29].

We call a system square if it has as many equations as unknowns. After adding linear equations to a square system, we [65] add slack variables in an embedding to make the overdetermined system square again. Using a flag of linear spaces, defined by a decreasing sequence of subsets of the $k$ general hyperplanes,

$$
L=L_{k} \supset L_{k-1} \supset \cdots \supset L_{1} \supset L_{0}=\emptyset,
$$

we move solutions with nonzero slack values to generic points on lower dimensional components, using a cascade of homotopies [65]. By default, the top dimension $k$ in the cascade starts at $n-1$, but if we know the system has only solution curves we start with $k=1$. According to [27], the dimension of an algebraic variety can be determined in polynomial time.

The cost of the homotopy cascade algorithm is determined by the number of paths, starting at the solutions of the top dimensional system. By the embedding, this top dimensional system has only isolated roots and thus one may apply any solver to compute those isolated roots. To count the number of solutions for square systems with generic coefficients we look at the Newton polytopes. The Newton polytopes are spanned by the exponents of the monomials which occur with nonzero coefficients in the system. We formally describe sparse polynomials as follows.

Definition 2.2 Given a polynomial

$$
f(\mathbf{x})=\sum_{\mathbf{a} \in A} c_{\mathbf{a}} \mathbf{x}^{\mathbf{a}}, \quad c_{\mathbf{a}} \neq 0, \quad \mathbf{x}^{\mathbf{a}}=x_{1}^{a_{1}} x_{2}^{a_{2}} \cdots x_{n}^{a_{n}},
$$

the support $A$ of $f$ collects only those exponent vectors with nonzero coefficient in $f$. The convex hull of $A$ is the Newton polytope of $f$. We denote the inner product by $\langle\cdot, \cdot\rangle$. To define faces $\partial_{\mathbf{v}} P$ of $P$ we use a support function $p$ :

$$
p(\mathbf{v})=\min _{\mathbf{x} \in P}\langle\mathbf{x}, \mathbf{v}\rangle \quad \text { so } \quad \partial_{\mathbf{v}} P=\{\mathbf{x} \in P \mid\langle\mathbf{x}, \mathbf{v}\rangle=p(\mathbf{v})\} .
$$

The equation $\langle\mathbf{x}, \mathbf{v}\rangle=p(\mathbf{v})$ determines a supporting hyperplane for the face $\partial_{\mathbf{v}} P$. A vector $\mathbf{v}$ perpendicular to a $k$-dimensional face $\partial_{\mathbf{v}} P$ of $P$ lies in an $(n-k)$-dimensional cone.

In defining support functions as in (3), we choose the minimum instead of the maximum convention, opting for inner rather than outer normals. These minimum and maximum conventions correspond to letting a deformation parameter respectively go to zero or to infinity. In [26], Newton polytopes arise as compactifications of amoebas [51], obtained by taking logarithms of the variety [7]. Algorithms to compute amoebas are presented in [74]. We refer to [75] and [82] for references on polytopes. For a system $\mathbf{f}(\mathbf{x})=\mathbf{0}$, we collect the Newton polytopes of the tuple of polynomials $\mathbf{f}=\left(f_{1}, f_{2}, \ldots, f_{n}\right)$ in the tuple $\mathbf{P}=\left(P_{1}, P_{2}, \ldots, P_{n}\right)$.

Definition 2.3 Given a tuple of Newton polytopes $\mathbf{P}=\left(P_{1}, P_{2}, \ldots, P_{n}\right)$ we define the mixed volume $V_{n}(\mathbf{P})$ via the formula

$$
V_{n}\left(P_{1}, P_{2}, \ldots, P_{n}\right)=\sum_{\mathbf{v} \in \mathbb{Z}^{n}} p_{1}(\mathbf{v}) V_{n-1}\left(\partial_{\mathbf{v}} P_{2}, \ldots, \partial_{\mathbf{v}} P_{n}\right),
$$


where $p_{1}$ is the support function for $P_{1}$ and $V_{1}$ is the length of a line segment. Vectors $\mathbf{v}$ are normalized so the components of $\mathbf{v}$ have their greatest common divisor equal to 1 .

The inductive formula in (4) for the mixed volume is explained in [13, §25.2] and in [64, Lemma 5.1.1]. Because polytopes are spanned by only finitely many points, only finitely many vectors $\mathbf{v}$ will yield a nonzero contribution to (4). In the special case: $P=P_{1}=P_{2}=\cdots=P_{n}$ : $V_{n}(\mathbf{P})=n$ !volume $(P)$. Handling the mixed case, when several but not all polytopes are repeated, is important for efficient algorithms, developed in [23], [47], and [53]. Mixed volumes are at the core of classical geometry [64] and have applications to tomography [25].

Theorem 2.4 (Bernshteǐn Theorem A [8]) The number of roots of a generic system equals the mixed volume of its Newton polytopes. For any system, the mixed volume bounds the number of isolated solutions in $\left(\mathbb{C}^{*}\right)^{n}, \mathbb{C}^{*}=\mathbb{C} \backslash\{0\}$.

In the same paper, Bernshtein gave in his second theorem precise conditions for the mixed volume to be sharp. Because of these conditions, we speak of count [14] instead of bound, see also $[62,63]$. We will formulate the second theorem later, but now we have introduced enough terminology to formulate our problem statement. Theorem 2.4 generalizes Kushnirenko's theorem [43] which considers systems where all polynomials have the same Newton polytope. The proof for Theorem 2.4 given in [8] is constructive (proofs by Khovanskiî are in [41, 42]) and served as basis for a polyhedral method in [80] to compute all isolated solutions. A more general polyhedral homotopy method was developed in [34], see also [72]. The authors of [39] address the complexity of Bernshtein's first theorem. The development of recent software for polyhedral homotopies is described in [32] and [44].

We list three problems with the current use of witness sets. First and foremost, as we add hyperplanes to the system, the polyhedral root count increases for sparse systems. For example, for a benchmark problem like the cyclic 8-roots system [9], adding one hyperplane raises the mixed volume from 2,560 to 4,176. For cyclic 12-roots, the same operation brings the mixed volume from 500,352 to 983,952. While the cascade will give us all solutions at the end, if we are only interested in the curves, we do not want to compute start solutions to the isolated roots. The second problem concerns symmetry. Many polynomial systems have obvious permutation symmetries and with polyhedral methods we can setup symmetric homotopies [78] to compute only the generators of each orbit of isolated roots. However, extending the symmetric polyhedral homotopies of [78] to deal with positive dimensional solution sets conflicts with the current witness set data representation. Thirdly, users of numerical algebraic geometry methods require and need guarantees for the results to be correct. Once we have a witness set, any path tracker may be used to sample points on the solution set. While path trackers are standard in numerical analysis, users unfamiliar or uncomfortable with floating-point computations require exact answers. Additionally, to determine the degree of a solution set correctly, we must be able to certify that all solutions have been found.

\section{Proper Algebraic Curves defined by Binomial Systems}

A binomial system has exactly two monomials with a nonzero coefficient in every equation. We consider $n-1$ equations in $n$ variables. By limiting the number of equations and restricting 
to nontrivial solutions in $\left(\mathbb{C}^{*}\right)^{n}$ we are reducing the complexity of the problem. However, even already for binomial systems, the complexity of counting all isolated solutions is \#P-complete [15].

For example $(n=3)$ :

$$
\left\{\begin{array} { r } 
{ x _ { 1 } x _ { 2 } ^ { 2 } x _ { 3 } - 2 x _ { 1 } ^ { 2 } x _ { 2 } ^ { 3 } x _ { 3 } = 0 } \\
{ 3 x _ { 1 } ^ { 2 } x _ { 2 } ^ { 2 } x _ { 3 } ^ { 5 } + 9 x _ { 1 } x _ { 2 } x _ { 3 } = 0 }
\end{array} \equiv \left\{\begin{array}{llll}
x_{1}^{-1} & x_{2}^{-1}= & 2 \\
x_{1}^{2} & x_{2} & x_{3}^{4}= & -3
\end{array}\right.\right.
$$

The system at the right of (5) is a normal form of the system. For general $n$, we can always write a binomial system as a tuple of equations of the form $\mathrm{x}^{\mathbf{a}}=c$. Writing a system in this normal form removes trivial solutions with zero coordinates. For a binomial system in its normal form, we collect all exponents in a matrix $A \in \mathbb{Z}^{(n-1) \times n}$ and its coefficients in $\mathbf{c} \in\left(\mathbb{C}^{*}\right)^{n-1}$. Continuing the example, we have:

$$
A=\left[\begin{array}{rrr}
-1 & -1 & 0 \\
2 & 1 & 4
\end{array}\right], \quad \operatorname{rank}(A)=2, \quad \mathbf{v}=\left[\begin{array}{r}
4 \\
-4 \\
-1
\end{array}\right]: A \mathbf{v}=\mathbf{0}
$$

The vector $\mathbf{v}$ in the kernel of $A$ will determine the shape of the solution curve.

For $A: \operatorname{rank}(A)=n-1$, there is a unique vector $\mathbf{v}$ in the kernel. A binomial system of $n-1$ equations in $n$ variables will have a proper (i.e.: not contained in any other higher dimensional solution set) solution curve in $\mathbb{C}^{*}$ if and only if the rank of the exponent matrix $A$ is $n-1$. Although a normal form of a binomial system is not unique, the vector $\mathbf{v}$ for a proper algebraic curve is independent of the choice of a particular normal form. We will show that proper algebraic curves have solutions of the type $x_{k}=c_{k} t^{v_{k}}$, with $c_{k} \in \mathbb{C}^{*}, k=1,2, \ldots, n$.

To simplify the system, we use $\mathbf{v}$ to define a unimodular matrix $M(\operatorname{det}(M)=1)$ and a coordinate transformation (called a power transformation in [12]) denoted by $\mathbf{x}=\mathbf{y}^{M}$ :

$$
M=\left[\begin{array}{lll}
+4 & 0 & 1 \\
-4 & 1 & 0 \\
-1 & 0 & 0
\end{array}\right] \quad A M=\left[\begin{array}{rrr}
0 & -1 & -1 \\
0 & 1 & 2
\end{array}\right] \quad\left\{\begin{array}{l}
x_{1}=y_{1}^{+4} y_{3} \\
x_{2}=y_{1}^{-4} y_{2} \\
x_{3}=y_{1}^{-1}
\end{array}\right.
$$

After applying the coordinate transformation, defined by $M$, we can divide out the variable $y_{1}$ and we obtain a system of two equations in two unknowns: $y_{2}$ and $y_{3}$. For our example, we find one solution

$$
\left(y_{2}=-\frac{1}{12}, y_{3}=-6\right) \quad \text { to }\left\{\begin{array}{lll}
y_{2}^{-1} & y_{3}^{-1}= & 2 \\
y_{2} & y_{3}^{2}= & -3 .
\end{array}\right.
$$

We rename the free variable $y_{1}$ to $t$ and use the values found for $y_{2}$ and $y_{3}$ in the representation of the solution curve, see the left of (9). To compute the degree of the curve, we add a random hyperplane and substitute the expression found for the solution:

$$
\left\{\begin{array} { l } 
{ x _ { 1 } = - 6 t ^ { + 4 } } \\
{ x _ { 2 } = - \frac { 1 } { 1 2 } t ^ { - 4 } } \\
{ x _ { 3 } = t ^ { - 1 } }
\end{array} \quad \left\{\begin{array}{l}
x_{1}^{-1} x_{2}^{-1}=2 \\
x_{1}^{2} x_{2} \quad x_{3}^{4}=-3 \\
\gamma_{0}+\gamma_{1} x_{1}+\gamma_{2} x_{2}+\gamma_{3} x_{3}=0
\end{array}\right.\right.
$$


The coefficients $\gamma_{0}, \gamma_{1}, \gamma_{2}$, and $\gamma_{3}$ are random complex numbers. After substitution the left of (9) into the right of (9) we obtain $\gamma_{0}+\gamma_{1}\left(-6 t^{+4}\right)+\gamma_{2}\left(-\frac{1}{12} t^{-4}\right)+\gamma_{3} t^{-1}$. Clearing denominators, we find a polynomial in $t$ of degree 8 .

Given the leading term of the Puiseux series expansion for an algebraic curve, the degree of the curve follows from the leading exponents of the Puiseux series. We can formalize this in the following proposition:

Proposition 3.1 Consider a proper algebraic curve defined by $\mathbf{x}^{A}=\mathbf{c}$, with $\mathbf{v}$ : $A \mathbf{v}=\mathbf{0}$. Let $M$ be the unimodular matrix in the transformation $\mathbf{x}=\mathbf{y}^{M}$ that eliminates $y_{1}$. Let $B$ be the matrix obtained from removing the first zero column of $A M$. Then the degree of the curve equals

$$
|\operatorname{det}(B)| \times\left|\max _{i=1}^{n} v_{i}-\min _{i=1}^{n} v_{i}\right| \text {. }
$$

Proof. The form of the solution curve is $x_{k}=\alpha_{k} t^{v_{k}}$ where the coefficients $\alpha_{k}$ are the nonzero roots of $\mathbf{y}^{A M}=\mathbf{c}$. The number of roots equals $|\operatorname{det}(B)|$. The curve will have as many components as $|\operatorname{det}(B)|$. Because every component has the same degrees in $t$, every component will have the same degree, so it suffices to compute the degree of one component.

To compute the degree of one component, we reduce it to as many isolated points as its degree, intersecting it with a hyperplane with random coefficients. Substituting the form of the solution component into that hyperplane yields a polynomial in $t$. To clear denominators we multiply by the most negative exponent or we divide out trivial solutions by subtracting the lowest positive exponent of $\mathbf{v}$.

We point out that the formula (10) is not invariant to unimodular transformations. Consider for example the plane curve defined by $f\left(x_{1}, x_{2}\right)=x_{1} x_{2}-1=0$ with $\mathbf{v}=(+1,-1)$. The unimodular coordinate transformation defined by $\mathbf{v}: x_{1}=y_{1}, x_{2}=y_{1}^{-1} y_{2}$ reduces $f$ to $y_{2}-1=0$. In the new $y$-coordinates, we now have a line $\left(y_{1}, 1\right)$.

For a proper algebraic curve defined by a binomial system we can provide an exact certificate for its degree, independently of the choice of the coefficients. The vector $\mathbf{v}$ we computed in the example above is an example of a tropism. We define tropisms for general polynomial systems in the next section.

Although the focus of this section is on proper algebraic curves defined by binomial systems, we sketch the generalization of Proposition 3.1 into an algorithm to compute the degree of any proper $k$-dimensional algebraic surface defined by a system $\mathbf{x}^{A}=\mathbf{c}$ of $n-k$ binomial equations. As the surface is proper, the kernel of $A$ has dimension $k$ and we first compute $k$ linearly independent lattice vectors $\mathbf{v}_{i} \in \mathbb{N}^{n}: A \mathbf{v}_{i}=\mathbf{0}, i=1,2, \ldots, k$. This basis for the kernel of $A$ must be reduced [31]. This computation leads to an explicit parametrization of the surface: $x_{j}=\alpha_{j} t_{1}^{v_{1 j}} t_{2}^{v_{2 j}} \cdots t_{k}^{v_{k j}}$, for some nonzero constants $\alpha_{j} \in \mathbb{C}^{*}, j=1,2, \ldots, n$. Using the witness set representation to compute the degree, we add $k$ general linear equations $L$ to the system. Substituting the explicit parameterization of the surface into $L$ leads to a system of $k$ polynomial equations in the variables $t_{i}, i=1,2, \ldots, k$. All $k$ equations have the same support, denote this support by $B$. The volume of the polytope spanned by $B$ gives the number of solutions of the system (Kushnirenko's theorem [43]) and thus the degree of the surface. This generalization could lead to an alternative proof of [71, Theorem 4.16] relating the degree of a variety defined by a toric ideal to the volume of a polytope. 


\section{Tropisms and Initial Forms}

Puiseux series occupy a central role in the study of algebraic curves. The leading exponents of the series are called tropisms, defined by the Newton polytopes of a polynomial system.

Definition 4.1 Consider $\mathbf{f}(\mathbf{x})=\mathbf{0}$ with Newton polytopes in $\left(P_{1}, P_{2}, \ldots, P_{N}\right)$. A tropism is a vector perpendicular to one edge of each $P_{i}$, for $i=1,2, \ldots, N$.

Our definition of tropisms differs from the usual one in the literature ([45], [49]) where all coordinates in the tropism (or critical tropism in singularity theory [45]) are required to be positive. This requirement is natural if one looks for one point on a solution curve in affine space and then shifts that point to the origin. The only normalization we will require is on the sign of the first coordinate. We will provide arguments for this normalization when we consider the asymptotics of witness sets in the next section. The other difference in the definition is then that we may have tropisms pointing out isolated solutions at infinity, solutions that do not give rise to an initial term in a Puiseux series expansion. Using a more refined terminology we could give the $\mathbf{v}$ in Definition 4.2 the name pretropism, as a part of a tropical prevariety. A tropical prevariety [11] corresponds to the intersection of the normal cones of the polytopes.

The edges perpendicular to a tropism are Newton polytopes of an initial form system which may have solutions in $\left(\mathbb{C}^{*}\right)^{n}$.

Definition 4.2 Let $\mathbf{v} \in \mathbb{Z}^{n} \backslash\{0\}$ and $f(\mathbf{x})=\sum_{\mathbf{a} \in A} c_{\mathbf{a}} \mathbf{x}^{\mathbf{a}}$. Denoting the inner product by $\langle\cdot, \cdot\rangle$, the initial form of $f$ in the direction $\mathbf{v}$ is

$$
\operatorname{in}_{\mathbf{v}} f(\mathbf{x})=\sum_{\substack{\mathbf{a} \in A \\\langle\mathbf{a}, \mathbf{v}\rangle=m}} c_{\mathbf{a}} \mathbf{x}^{\mathbf{a}} \text { with } m=\min \{\langle\mathbf{a}, \mathbf{v}\rangle \mid \mathbf{a} \in A\} .
$$

Let $\mathbf{f}=\left(f_{1}, f_{2}, \ldots, f_{N}\right)$ be a tuple of polynomials. For $\mathbf{v} \in \mathbb{Z}^{n} \backslash\{0\}$, the initial form system $\operatorname{in}_{\mathbf{v}} \mathbf{f}(\mathbf{x})=\mathbf{0}$ is defined by the tuple $\operatorname{in}_{\mathbf{v}} \mathbf{f}=\left(\operatorname{in}_{\mathbf{v}} f_{1}, \operatorname{in}_{\mathbf{v}} f_{2}, \ldots, \operatorname{in}_{\mathbf{v}} f_{N}\right)$.

The intial form systems of interest to us are those defined via tropisms. As tropisms are vectors perpendicular to edges of the Newton polytopes, the number of points in the support of each initial form is then at least - but not exactly — two. Although the initial form system is thus not necessarily a binomial system, we can always eliminate one variable.

In [12] and [40], systems supported on faces of Newton polytopes are called truncated systems. This terminology refers to the process of substituting the power series and then selecting those terms that correspond to the lowest power in the variable of the series. We prefer to use initial forms because of the relationship with term orders widely used for Gröbner bases [71], [75].

Of special importance are the conditions for which the mixed volume is sharp, formulated in the tropical language.

Theorem 4.3 (Bernshteǐn Theorem B [8]) Consider $\mathbf{f}(\mathbf{x})=\mathbf{0}, \mathbf{f}=\left(f_{1}, f_{2}, \ldots, f_{n}\right), \mathbf{x}=$ $\left(x_{1}, x_{2}, \ldots, x_{n}\right)$. If for all tropisms $\mathbf{v}: \operatorname{in}_{\mathbf{v}} \mathbf{f}(\mathbf{x})=\mathbf{0}$ has no solutions in $\left(\mathbb{C}^{*}\right)^{n}$, then $\mathbf{f}(\mathbf{x})=\mathbf{0}$ has exactly as many isolated solutions in $\left(\mathbb{C}^{*}\right)^{n}$ as $V_{n}(\mathbf{P})$. 
If there is a positive dimensional solution set, then this set stretches out to infinity and the system $f(\mathbf{x})=\mathbf{0}$ must have solutions at infinity. Of particular interest is the following consequence of Bernshteinn's second theorem.

Corollary 4.4 If there are no tropisms $\mathbf{v}$ for which $\operatorname{in}_{\mathbf{v}} \mathbf{f}(\mathbf{x})=\mathbf{0}$ has roots in $\left(\mathbb{C}^{*}\right)^{n}$ then $\mathbf{f}(\mathbf{x})=\mathbf{0}$ has no solutions at infinity.

The tropisms and the roots of the corresponding initial form systems give the leading term of the Puiseux series expansion of the solution curve. The second term in the Puiseux series expansion will provide the certificate for the existence of the solution curve. Also the coefficients of the second term will be roots of a truncated polynomial system. Once we have those roots, we can further grow the Puiseux series expansion symbolically, or apply numerical predictor-corrector methods to sample points along the solution curve. In Figure 4 (slightly adapted from [1]) we sketch the idea for computing this certificate. Recall that the proper in proper algebraic curve also means regular, i.e.: free of multiplicities. Puiseux series at multiple roots may coincide for many more terms past the second term.

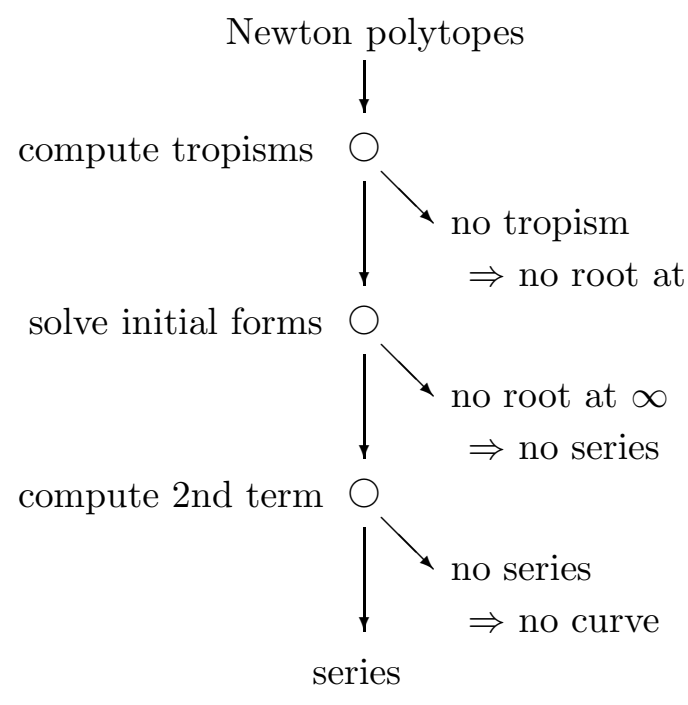

Figure 1: Computing a certificate for a proper algebraic curve.

The computation of the second term in the Puiseux series goes along the so-called NewtonPuiseux method, as outlined in the proof of the theorem of Puiseux [81], see also [19]. In [2] algorithms for Puiseux series for space curves are described and an implementation in CoCoA is mentioned. General fractional power series solutions are described in [50]. See [38], [39] and [59] for recent symbolic algorithms, and [57], [58] for a symbolic-numeric approach.

Algebraic curves defined by binomial systems can be solved by one tropism but it may happen that one tropism solves a more general system. Consider for example the cyclic 4-roots problem.

$$
\mathbf{f}(\mathbf{x})=\left\{\begin{array}{c}
x_{1}+x_{2}+x_{3}+x_{4}=0 \\
x_{1} x_{2}+x_{2} x_{3}+x_{3} x_{4}+x_{4} x_{1}=0 \\
x_{1} x_{2} x_{3}+x_{2} x_{3} x_{4}+x_{3} x_{4} x_{1}+x_{4} x_{1} x_{2}=0 \\
x_{1} x_{2} x_{3} x_{4}-1=0
\end{array}\right.
$$


There is one tropism $\mathbf{v}=(1,-1,1,-1)$ which leads to the initial form $\operatorname{system~}_{\operatorname{in}} f(\mathbf{z})=\mathbf{0}$ :

$$
\operatorname{in}_{\mathbf{v}} \mathbf{f}(\mathbf{x})=\left\{\begin{array} { c } 
{ x _ { 2 } + x _ { 4 } = 0 } \\
{ x _ { 1 } x _ { 2 } + x _ { 2 } x _ { 3 } + x _ { 3 } x _ { 4 } + x _ { 4 } x _ { 1 } = 0 } \\
{ x _ { 2 } x _ { 3 } x _ { 4 } + x _ { 4 } x _ { 1 } x _ { 2 } = 0 } \\
{ x _ { 1 } x _ { 2 } x _ { 3 } x _ { 4 } - 1 = 0 }
\end{array} \quad \left\{\begin{array}{l}
x_{1}=y_{1} \\
x_{2}=y_{1}^{-1} y_{2} \\
x_{3}=y_{1} y_{3} \\
x_{4}=y_{1}^{-1} y_{4}
\end{array}\right.\right.
$$

The system $\operatorname{in}_{\mathbf{v}} \mathbf{f}(\mathbf{y})=\mathbf{0}$ has two solutions and we find the two solution curves: $\left(t,-t^{-1},-t, t^{-1}\right)$ and $\left(t, t^{-1},-t,-t^{-1}\right)$.

Note that $\mathbf{v}=(-1,1,-1,1)$ is a tropism as well for the solution curves of cyclic 4-roots, but considering this tropism corresponds to setting $x_{1}=t^{-1}$ or moving the curve to infinity instead of to zero as $t$ goes to zero. We will examine this in greater generality in the next section.

\section{$5 \quad$ Asymptotics of Witness Sets}

One way to compute tropisms would be to start from a witness set for an algebraic curve in $n$-space given by $d$ points on a general hyperplane $c_{0}+c_{1} x_{1}+c_{2} x_{2}+\cdots+c_{n} x_{n}=0$ and satisfying a system $\mathbf{f}(\mathbf{x})=\mathbf{0}$. We then deform a witness set for a curve in two stages:

1. The first homotopy moves to a hyperplane in special position:

$$
\mathbf{h}(\mathbf{x}, t)=\left\{\begin{array}{c}
\mathbf{f}(\mathbf{x})=\mathbf{0} \\
\left(c_{0}+c_{1} x_{1}+\cdots+c_{n} x_{n}\right) t+\left(c_{0}+c_{1} x_{1}\right)(1-t)=0, \text { for } t \text { from } 1 \text { to } 0 .
\end{array}\right.
$$

2. After renaming $c_{0}+c_{1} x_{1}=0$ into $x_{1}=\gamma$, we let $x_{1}$ go to zero with the following homotopy:

$$
\mathbf{h}(\mathbf{x}, t)=\left\{\begin{array}{c}
\mathbf{f}(\mathbf{x})=\mathbf{0} \\
x_{1}-\gamma t=0, \text { for } t \text { from } 1 \text { to } 0
\end{array}\right.
$$

The two homotopies need further study. In the first homotopy (14) some paths will diverge, consider for example $f\left(x_{1}, x_{2}\right)=x_{1} x_{2}-1$. Even all paths may diverge if the solution curve lies in some hyperplane perpendicular to the first coordinate axis $x_{1}=c$ with $c$ different from $-c_{0} / c_{1}$.

Lemma 5.1 All solutions at the end of the homotopy $\mathbf{h}(\mathbf{x}, t)=\mathbf{0}$ of (14) lie on the curve defined by $\mathbf{f}(\mathbf{x})=\mathbf{0}$ and in the hyperplane $x_{1}=-c_{0} / c_{1}$.

Proof. We claim that we find the same solutions to $\mathbf{h}(\mathbf{x}, t=0)=\mathbf{0}$ either by using the homotopy in (14) or by solving $\mathbf{h}(\mathbf{x}, 0)=\mathbf{0}$ directly. This claim follows from cheater's homotopy [48] or the more general coefficient-parameter polynomial continuation [55].

The second claim we make in the main theorem below is that we recover all data lost with tropisms. For simplest example of the hyperbola $x_{1} x_{2}-1=0$ : its solution is $\left(x_{1}=t, x_{2}=t^{-1}\right)$ and the tropism is $\mathbf{v}=(1,-1)$. The lemma below extends the normal form for the Puiseux series expansion for plane curves (as used in [81]) to general space curves. 
Lemma 5.2 Ast $\rightarrow 0$ in the homotopy (15), the leading powers of the Puiseux series expansions are the components of a tropism. In particular, the expansions have the form

$$
\left\{\begin{array}{l}
x_{1}=t \\
x_{k}=c_{k} t^{v_{k}}(1+O(t)), \quad k=2, \ldots, n .
\end{array}\right.
$$

Proof. Following Bernshtern's second theorem, a solution at infinity is a solution in $\left(\mathbb{C}^{*}\right)^{n}$ of an initial form system. For a solution to have values in $\left(\mathbb{C}^{*}\right)^{n}$, all equations in that system need to have at least two monomials. So the system is an initial form system defined by a tropism. To arrive at the form of (16) for the solution defined by the homotopy (15) we rescale the parameter $t$ so we may replace $x_{1}=\gamma t$ by $x_{1}=t$.

Also in the second homotopy, solution paths are most likely to diverge and the directions of the diverging paths are defined by the tropisms. The numerical computation of those directions can be done by endgames using power series as in [56] and [35].

Definition 5.3 Given a system $\mathbf{f}(\mathbf{x})=\mathbf{0}$ which defines a proper algebraic curve. Consider a Puiseux series expansion of the form

$$
\left\{\begin{array}{l}
x_{1}=t \\
x_{k}=c_{k} t^{v_{k}}+d_{k} t^{w_{k}}+\cdots \quad c_{k}, d_{k} \in \mathbb{C}^{*}, v_{k}, w_{k} \in \mathbb{Q}, v_{k}<w_{k}, k=2, \ldots, n .
\end{array}\right.
$$

Then a certificate for the solution curve consist of the exponents $\left(v_{2}, \ldots, v_{n}\right),\left(w_{2}, \ldots, w_{n}\right) \in \mathbb{Q}^{n-1}$ and the coefficients $\left(c_{2}, \ldots, c_{n}\right),\left(d_{2}, \ldots, d_{n}\right) \in\left(\mathbb{C}^{*}\right)^{n-1}$.

The tropism $\mathbf{v}$ (or pretropism) shows there are solutions at infinity, but solutions at infinity could be isolated. In that case the tropism $\mathbf{v}$ is still a certificate (but then more like a death certificate) for the lack of sharpness of the mixed volume to count all isolated roots. The exponents $\mathbf{w}$ of the second term in the series show the solution is part of a curve. Arguing in favor of extending the term tropism rather than reserving it only for the leading exponents in the series, we point out that a certificate consists of a pair of vectors: $(\mathbf{v}, \mathbf{w})$ and a corresponding pair of solutions $(\mathbf{c}, \mathbf{d})$, respectively of an initial form system and a truncated system. As solutions of overdetermined polynomial systems, the coefficients $(\mathbf{c}, \mathbf{d})$ can be certified [20] by $\alpha$-theory [10].

Relating the data $(\mathbf{v}, \mathbf{w})$ and $(\mathbf{c}, \mathbf{d})$ to a witness set, we note that the data corresponds geometrically to cutting the curve with a special hyperplane $x_{1}=0$. There is the risk of missing curves in coordinate hyperplane $x_{1}=c$, for $c \in \mathbb{C}^{*}$ and there may also be singular solutions occurring for $x_{1}=0$. Except for these two difficulties, the certificate provides a predictor to sample the solution curve. The capability to sample a solution curve is intrinsic in the definition of a witness set, but to verify this capability one needs to compute at least one step of Newton's method at at least one point of the witness set. Substituting the certificate in the original system — with and without the second term and watching the degree in $t$ increase - is a more elementary operation.

On the one hand, we can view the certificate as a very special witness set, obtained by intersecting the curve with the hyperplane $x_{1}=0$. On the other hand, we can view the certificate as a very special lifting fiber, where one free variable $x_{1}$ is specialized to zero. 
Lemma 5.4 The certificate of (17) may be written in the form

$$
\begin{cases}x_{1}=t^{\nu_{1}}, & \nu_{1} \geq 1, \\ x_{k}=\alpha_{k} t^{\nu_{k}}+\beta_{k} t^{\mu_{k}}, & k=2, \ldots, n,\end{cases}
$$

where $\alpha_{k}, \beta_{k} \in \mathbb{C}^{*}$ and $\nu_{k}, \mu_{k} \in \mathbb{Z}$ and $\nu_{1}$ is the smallest natural number to clear the denominators in the series for the other components $x_{k}, k>1$. Then the degree of the branch is determined by

$$
\# R \times\left|\max _{i=1}^{n} \nu_{i}-\min _{i=1}^{n} \nu_{i}\right|
$$

where $R$ is the set of initial roots of the initial form system $\operatorname{in}_{\boldsymbol{\nu}} \mathbf{f}\left(\mathbf{x}=\mathbf{y}^{M}\right)=\mathbf{0}$, for $M$ a unimodular matrix with first column equal to $\boldsymbol{\nu}=\left(\nu_{1}, \nu_{2}, \ldots, \nu_{n}\right)$.

Proof. The existence of $\nu_{1}$ follows from the definition of Puiseux expansions. Suppose there would not be a smallest $\nu_{1}$ to clear denominators, then we could make a plane curve for which we could not clear denominators. Proposition 3.1 is generalized in two ways:

1. The initial form system $\operatorname{in}_{\boldsymbol{\nu}} \mathbf{f}(\mathbf{y})=\mathbf{0}$ is no longer a binomial system for which we can count the number of roots via a determinant. Instead we now solve $\operatorname{in}_{\boldsymbol{\nu}} \mathbf{f}(\mathbf{y})=\mathbf{0}$ and collect the roots in $R$.

2. We have to show that only the leading terms in the Puiseux series expansion determine the degree of the solution branch. Consider $t \rightarrow 0$ and consider the solutions in the hyperplane $x_{1}=t^{\nu_{1}}$. By the form of the expansion we cannot have more solutions for $t>0$ then we would have at $t=0$. At $t=0$, only the leading terms matter in defining the initial form

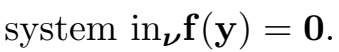

The key point of the two homotopies we considered above is the argument for the normalization of the first coordinate $v_{1}$ of the tropisms to $v_{1}>0$. Moving the degree many points on the curve on the hyperplane $x_{1}=\gamma$ to $x_{1}=0$ with $x=t$ is equivalent to moving those points to infinity with $x=t^{-1}$. By equivalent we mean that we do not obtain any new information about the curve by considering also tropisms with first coordinate of the opposite sign.

Theorem 5.5 Given a proper algebraic curve defined by a polynomial system $\mathbf{f}(\mathbf{x})=\mathbf{0}$ of $n$ equations in $n$ unknowns. Assume that the curve does not lie in a coordinate hyperplane perpendicular to the first coordinate axis. Then the degree of the curve corresponds to the number of certificates times the degree of each certificate.

Proof. If we consider the two asymptotic homotopies in sequence, then we may lose solutions in the first move, by putting the cutting hyperplane perpendicular to the first coordinate axis, while in the tropisms found in the second stage lead then to higher degrees. The claim of the theorem is to show that a potential loss of witness points is made up by the increase in the degree of the Puiseux series at the very end.

Executing the asymptotic homotopies in sequence is equivalent to combine them into one homotopy:

$$
\left\{\begin{array}{c}
\mathbf{f}(\mathbf{x})=\mathbf{0} \\
x_{1}+t\left(c_{0}+c_{2} x_{2}+\cdots+c_{n} x_{n}\right)=0 \quad \text { for } t \text { from } 1 \text { to } 0 .
\end{array}\right.
$$


Observe that the Newton polytopes of $\mathbf{f}$ are not deformed by this homotopy. So the structure of the space at infinity remains invariant as well. Applying polyhedral endgames [35] (see also [56]), the witness points will end at roots of initial form systems. So every witness point corresponds to one initial root and we cannot have more witness points than we have initial roots.

Using a weighted projective space [16], [17], [77], determined by the tropisms, we can extend the initial roots to roots for $t>0$. So the initial roots give rise to witness points.

The complexity of the certificate is mainly determined by the size of the initial form systems. In the best case, the initial form systems are binomial, while in the worst case the number of monomials in the initial form systems is of the same order of magnitude as the original system. But even in this worst case, the number of variables drops by one and this drop may be enough to get a more tractable problem.

We end this section sketching how to generalize the asymptotics of witness sets for any $k$ dimensional surface. The first homotopy move of (14) is generalized by deforming the $k$ general hyperplanes to $x_{i}=\gamma_{i}$, for random constants $\gamma_{i} \in \mathbb{C}^{*}, i=1,2, \ldots, k$. The deformation in the second homotopy (15) then becomes defined by $x_{i}=\gamma_{i} t$, for $t$ going from 1 to 0 . The asymptotics, as t approaches 0 , are then described by a Puiseux series that starts out with $x_{i}=t$, for $i=1,2, \ldots, k$ and continues with regular Puiseux series expansion for the remaining $n-k$ variables. The leading powers of these Puiseux series expansions (or the tropisms) then have their first $k$ components positive. While tropisms for curves are unique up to scaling, tropisms for $k$-dimensional surfaces span a $k$-dimensional polyhedral cone. A certificate would then start with a reduced lattice basis for this cone.

\section{Tropisms and Mixed Volumes}

Degenerating witness sets is an effective but not an efficient way to compute tropisms. Relating to mixed volumes, we indicate how to compute the tropisms directly from the Newton polytopes. Before we explain the lifting algorithm to compute mixed volumes we point out that Gfan [37] using the algorithms of [11] is more appropriate to compute tropisms. Our point of relating tropisms to mixed volumes is to investigate the connection between the generic number of isolated roots and the degrees of the solution curves of sparse polynomial systems.

Let $A=\left(A_{1}, A_{2}, \ldots, A_{n}\right)$ be the supports of $\mathbf{f}(\mathbf{x})=\mathbf{0}$. Following [21], [34], we summarize the mixed volume computation in three stages:

1. Lift $\mathbf{a} \in A_{i}$ using a function $\omega: \mathbb{Z}^{n} \rightarrow \mathbb{Z}: \mathbf{a} \mapsto \omega(\mathbf{a})$ and denote $\omega\left(A_{i}\right)=\{(\mathbf{a}, \omega(\mathbf{a})) \mid \mathbf{a} \in$ $\left.A_{i}\right\} \subseteq \mathbb{Z}^{n+1}, i=1,2, \ldots, n$.

2. The facets on the lower hull of the Minkowski sum $\sum_{i=1}^{n} \omega\left(A_{i}\right)$ spanned by one edge of each of $\omega\left(A_{i}\right)$ define mixed cells $C$.

3. The mixed volume is $V_{n}(A)=\sum_{C \subseteq A} \operatorname{Vol}(C)$.

$C$ is mixed 
By duality [79], mixed cells are defined by inner normals perpendicular to edges of the polytopes. These inner normals are tropisms with positive last coordinate.

Polyhedral homotopies [34] follow the computation of the mixed cells and the lifting function defines the powers of the new parameter $t$. In particular, polynomials are lifted as

$$
f(\mathbf{x})=\sum_{\mathbf{a} \in A} c_{\mathbf{a}} \mathbf{x}^{\mathbf{a}} \rightarrow \widehat{f}(\mathbf{x}, t)=\sum_{\mathbf{a} \in A} c_{\mathbf{a}} \mathbf{x}^{\mathbf{a}} t^{\omega(\mathbf{a})}
$$

using the same lifting function $\omega$ as before.

Now we look for solution curves of the form (16). We observe that since $x_{1}=t$, as lifting function we define $\tau$ as $\tau(\mathbf{a})=\operatorname{deg}\left(\mathbf{x}^{\mathbf{a}}, x_{1}\right)=a_{1}$ as lifting function. The lifting $\tau$ will work for systems of $n-1$ equations in $n$ variables. However, for systems with $n$ equations, if we use $t=x_{1}$, then we will have too few variables. Therefore, we use a slack variable $z$ in the lifting, and define $\tau$ as

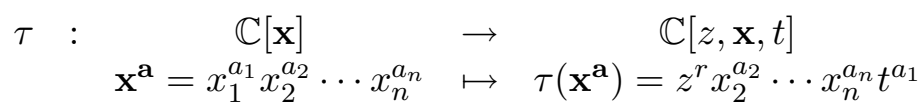

where $r$ is some random exponent. For exponent vectors a, we define $\tau$ as $\tau\left(a_{1}, a_{2}, \ldots, a_{n}\right)=$ $\left(r, a_{2}, \ldots, a_{n}, a_{1}\right)$. Note that $r=\omega(\mathbf{a})$, the usual random lifting used to calculate mixed volumes. If we take $t=x_{1}, z=t, r=\omega(\mathbf{a})$, then we get $t^{\omega(\mathbf{a})} x_{2}^{a_{2}} \cdots x_{n}^{a_{n}} x_{1}^{a_{1}}=\mathbf{x}^{\mathbf{a}} t^{\omega(\mathbf{a})}$, which is the random lifting commonly used in polyhedral homotopies [34]. With the slack variable we have again as many variables as equations and we can apply our mixed volume calculators.

Consider for example the following system:

$$
\mathbf{f}(\mathbf{x})=\left\{\begin{array}{rl}
x_{1} x_{3}-x_{1}-x_{3}+1 & =0 \\
x_{2}-1 & =0 \\
x_{3}-\gamma & =0
\end{array} \quad \gamma \in \mathbb{C}^{*} .\right.
$$

By elimination of $x_{2}$ and $x_{3}$ using the last two equations, we obtain one as the mixed volume of the Newton polytopes spanned by the supports of the polynomials in $\mathbf{f}$. This calculation also illustrates the inductive formula (4). Except for $\gamma=1$, the system has only one isolated solution: $(1,1, \gamma)$. For $\gamma=1$, the tropism $\mathbf{v}=(1,0,0)$ gives the exponents of $t$ in the solution line $\left(x_{1}=t, x_{2}=1, x_{3}=1\right)$. A witness set representation for this system needs to store two solutions: one solution for the witness set of the line in case $\gamma=1$ and one solution for the path leading to the isolated root in case $\gamma \neq 1$. To compute the tropism, we apply the map $\tau$ to every monomial in $\mathbf{f}$, for example:

$$
\tau(\mathbf{f})(z, \mathbf{x}, t)=\left\{\begin{aligned}
z^{8} x_{3} t-z^{5} t-x_{3}+1 & =0 \\
x_{2}-1 & =0 \\
x_{3}-\gamma & =0 .
\end{aligned}\right.
$$

The exponents 8 and 5 of the slack variable $z$ are chosen at random. The inner normal of interest is $\mathbf{v}=(0,0,0,1)$. The corresponding initial form system $\operatorname{in}_{\mathbf{v}}(\tau(\mathbf{f}))$ leaves only terms in $x_{2}$ and $x_{3}$. Only if $\gamma=1$ does $\operatorname{in}_{\mathbf{v}}(\tau(\mathbf{f}))\left(0, x_{2}, x_{3}, 0\right)=\mathbf{0}$ have a solution.

We claim that the tropisms to proper algebraic curves are in one-to-one correspondence with those inner normals to the mixed cells of the mixed subdivision induced by the lifting $\omega$ for which the $z$-component is zero. 
Proposition 6.1 Let $S$ be the set of mixed cells for an $n$-tuple $A$ in $n$-space lifted with $\tau$, introducing a slack variable $z$. Then $\mathbf{v}=\left(\mathbf{v}_{z}, \mathbf{v}_{\mathbf{x}}\right)$ is a tropism if and only if $\mathbf{v}_{z}=0$.

Proof. Denoting the inner normals to the cells in $S$ as $\mathbf{v}=\left(\mathbf{v}_{z}, \mathbf{v}_{\mathbf{x}}\right)$, points $(\mathbf{a}, \mathbf{b})$ in each cell satisfy

$$
r_{\mathbf{a}} \mathbf{v}_{z}+\mathbf{a}_{\mathbf{x}} \mathbf{v}_{\mathbf{x}}=r_{\mathbf{b}} \mathbf{v}_{z}+\mathbf{b}_{\mathbf{x}} \mathbf{v}_{\mathbf{x}}, \quad \mathbf{a}=\left(r_{\mathbf{a}}, \mathbf{a}_{\mathbf{x}}\right), \mathbf{b}=\left(r_{\mathbf{b}}, \mathbf{b}_{\mathbf{x}}\right) .
$$

We have to show two things:

1. Each inner normal $\mathbf{v}$ with $\mathbf{v}_{z}=0$ is a tropism.

If $\mathbf{v}_{z}=0$, then $\mathbf{a}_{\mathbf{x}} \mathbf{v}_{\mathbf{x}}=\mathbf{b}_{\mathbf{x}} \mathbf{v}_{\mathbf{x}}$ holds and the tropism is $\mathbf{v}_{\mathbf{x}}$ because $\left(\mathbf{a}_{\mathbf{x}}, \mathbf{b}_{\mathbf{x}}\right)$ span an edge of one of the Newton polytopes.

2. For every tropism we must have that $\mathbf{v}_{z}=0$.

Given a tropism $\mathbf{v}_{\mathbf{x}}$, we have $\mathbf{a}_{\mathbf{x}} \mathbf{v}_{\mathbf{x}}=\mathbf{b}_{\mathbf{x}} \mathbf{v}_{\mathbf{x}}$. Rewriting (25) to solve for $\mathbf{v}_{z}$ leads to

$$
\mathbf{v}_{z}\left(r_{\mathbf{a}}-r_{\mathbf{b}}\right)=\mathbf{b}_{\mathbf{x}} \mathbf{v}_{\mathbf{x}}-\mathbf{a}_{\mathbf{x}} \mathbf{v}_{\mathbf{x}}=0 \text {. }
$$

Since $r_{\mathbf{a}}$ and $r_{\mathbf{b}}$ are random numbers, $\mathbf{v}_{z}=0$.

Although this is already a more efficient method than applying polyhedral endgames in the asymptotic homotopies on witness sets, for practical purposes one would include the constraint $v_{z}=0$ already immediately in all feasibility tests the mixed volume calculator does. Ultimately, unlike the lift-and-prune approach [21] for mixed volume computation, the complexity of the problem of computing tropisms is governed by the shape of the polytopes and the relative position of the polytopes with respect to each other. Tropisms lie in the common refinement of cones of inner normals to faces of the Newton polytopes and algorithms of [11] as implemented in Gfan [37] are recommended.

\section{Preliminary Computational Experiments}

The cyclic $n$-roots problem is a widely known benchmark for polynomial system solvers, see e.g. [18], [22] and [47]. For those $n$ which are divisible by a square (e.g.: $n=4,8,9,12$ ), the system is known to have positive dimensional solution sets [3]. The general recipe to formulate the polynomial equations for any $n$ in this family is obvious from the cyclic 4-roots system, given above in (12).

The permutation symmetry in cyclic $n$-roots is generated by two elements $\left(x_{1}, x_{2}, x_{3}, \ldots, x_{n}\right)$ $\rightarrow\left(x_{2}, x_{3}, \ldots, x_{n}, x_{1}\right)$ and $\left(x_{1}, x_{2}, \ldots, x_{n-1}, x_{n}\right) \rightarrow\left(x_{n}, x_{n-1}, \ldots, x_{2}, x_{1}\right)$. We see these two generators as reading the solution vector once forwards and once backwards. This implies that one cyclic $n$-root can generate an orbit of up to $2 n$ solutions. Orbits of roots with repeated components are smaller in size. The same permutation group applies to the Newton polytopes one observation of [78] was that even if the system is not invariant under permutation symmetries, the polytopes often are - and thus also the tropisms. For every orbit of tropisms we have to solve the initial form system of only one generator. 
The numerical computations reported below were done with PHCpack [76], using a modified lifting in MixedVol [24]. An alternative mixed volume calculator is DEMiCs [52]. For the symbolic manipulations, Maple 11 was used. We emphasize that the computations reported below are preliminary, mainly to illustrate the concepts.

\section{1 cyclic 8-roots}

The program computed 29 tropisms, listed below in Table 1 and Table 2.

\begin{tabular}{r|rrr|rr|r|rrr|rrr}
3 & 1 & 1 & 1 & 1 & 1 & 1 & 1 & 1 & 1 & 1 & 1 & 1 \\
-1 & 1 & 1 & -3 & 1 & -1 & -1 & -1 & 0 & -1 & 0 & -1 & -1 \\
-1 & 1 & -3 & 1 & -1 & -1 & 1 & 1 & -1 & 1 & -1 & 1 & 0 \\
-1 & -3 & 1 & 1 & -1 & 1 & -1 & 0 & 0 & -1 & 1 & -1 & 1 \\
3 & 1 & 1 & 1 & 1 & 1 & 1 & -1 & 1 & 1 & -1 & 0 & 0 \\
-1 & 1 & 1 & -3 & 1 & -1 & -1 & 0 & -1 & 0 & 1 & 1 & -1 \\
-1 & 1 & -3 & 1 & -1 & -1 & 1 & 1 & 1 & -1 & -1 & 0 & 1 \\
-1 & -3 & 1 & 1 & -1 & 1 & -1 & -1 & -1 & 0 & 0 & -1 & -1
\end{tabular}

Table 1: First list of 13 tropisms for cyclic 8-roots, separated in 6 orbits.

$$
\begin{array}{rr|rr|rr|rr|rrr|rr|rr|rr}
1 & 1 & 1 & 1 & 1 & 1 & 1 & 1 & 1 & 1 & 1 & 1 & 1 & 1 & 1 & 1 \\
0 & -1 & 0 & -1 & 0 & -1 & 0 & -1 & 0 & -1 & 0 & -1 & 0 & -1 & 0 & 0 \\
0 & 1 & 0 & 0 & 0 & 1 & 0 & 0 & 0 & 0 & -1 & 1 & -1 & 0 & -1 & -1 \\
0 & 0 & 0 & 1 & -1 & 0 & -1 & 1 & -1 & 0 & 0 & 0 & 1 & 0 & 0 & 1 \\
-1 & 0 & -1 & 0 & 0 & 0 & 0 & 0 & 1 & 1 & 0 & -1 & -1 & 0 & 0 & 0 \\
0 & 0 & 1 & 0 & 0 & -1 & 1 & 0 & -1 & 0 & 0 & 0 & 0 & 1 & 1 & -1 \\
1 & -1 & -1 & 0 & 1 & 0 & -1 & -1 & 0 & 0 & 1 & 0 & 0 & 0 & 0 & 0 \\
-1 & 0 & 0 & -1 & -1 & 0 & 0 & 0 & 0 & -1 & -1 & 0 & 0 & -1 & -1 & 0
\end{array}
$$

Table 2: Second list of 16 tropisms for cyclic 8-roots, separated in 8 orbits.

The last tropism from Table $2: \mathbf{v}=(1,0,-1,1,0,-1,0,0)$, the initial form system is

$$
\operatorname{in}_{\mathbf{v}} \mathbf{f}(\mathbf{x})=\left\{\begin{array}{r}
x_{3}+x_{6}=0 \\
x_{2} x_{3}+x_{5} x_{6}+x_{6} x_{7}=0 \\
x_{5} x_{6} x_{7}+x_{6} x_{7} x_{8}=0 \\
x_{3} x_{4} x_{5} x_{6}+x_{5} x_{6} x_{7} x_{8}=0 \\
x_{2} x_{3} x_{4} x_{5} x_{6}+x_{3} x_{4} x_{5} x_{6} x_{7}=0 \\
x_{2} x_{3} x_{4} x_{5} x_{6} x_{7}+x_{3} x_{4} x_{5} x_{6} x_{7} x_{8}+x_{6} x_{7} x_{8} x_{1} x_{2} x_{3}=0 \\
x_{2} x_{3} x_{4} x_{5} x_{6} x_{7} x_{8}+x_{5} x_{6} x_{7} x_{8} x_{1} x_{2} x_{3}=0 \\
x_{1} x_{2} x_{3} x_{4} x_{5} x_{6} x_{7} x_{8}-1=0
\end{array}\right.
$$

To reduce the system into a simpler form, we perform the coordinate transformation, dividing 
out $y_{1}$ in the initial form system:

$$
\left\{\begin{array}{l}
x_{1}=y_{1} \\
x_{2}=y_{1}^{0} y_{2} \\
x_{3}=y_{1}^{-1} y_{3} \\
x_{4}=y_{1}^{1} y_{4} \\
x_{5}=y_{1}^{0} y_{5} \\
x_{6}=y_{1}^{-1} y_{6} \\
x_{7}=y_{1}^{0} y_{7} \mathbf{f}(\mathbf{y})=\left\{\begin{array}{rl}
y_{3}+y_{6} & =0 \\
x_{8} & =y_{1}^{0} y_{8}
\end{array} \quad y_{2} y_{3}+y_{5} y_{6}+y_{6} y_{7}=0\right. \\
y_{5} y_{6} y_{7}+y_{6} y_{7} y_{8}=0 \\
y_{3} y_{4} y_{5} y_{6}+y_{5} y_{6} y_{7} y_{8}=0 \\
y_{2} y_{3} y_{4} y_{5} y_{6}+y_{3} y_{4} y_{5} y_{6} y_{7}=0 \\
y_{2} y_{3} y_{4} y_{5} y_{6} y_{7}+y_{3} y_{4} y_{5} y_{6} y_{7} y_{8}+y_{6} y_{7} y_{8} y_{2} y_{3}=0 \\
y_{2} y_{3} y_{4} y_{5} y_{6} y_{7} y_{8}+y_{5} y_{6} y_{7} y_{8} y_{2} y_{3}=0 \\
y_{2} y_{3} y_{4} y_{5} y_{6} y_{7} y_{8}-1=0
\end{array}\right.
$$

To solve the overconstrained initial form, we introduce a slack variable $s$ and generate eight random numbers $\gamma_{k} \in \mathbb{C}^{*}, k=1,2, \ldots, 8$ to multiply $s$ with. Then we solve

$$
\operatorname{in}_{\mathbf{v}} \mathbf{f}(\mathbf{y}, s)=\left\{\begin{array}{r}
y_{3}+y_{6}+\gamma_{1} s=0 \\
y_{2} y_{3}+y_{5} y_{6}+y_{6} y_{7}+\gamma_{2} s=0 \\
y_{5} y_{6} y_{7}+y_{6} y_{7} y_{8}+\gamma_{3} s=0 \\
y_{3} y_{4} y_{5} y_{6}+y_{5} y_{6} y_{7} y_{8}+\gamma_{4} s=0 \\
y_{2} y_{3} y_{4} y_{5} y_{6}+y_{3} y_{4} y_{5} y_{6} y_{7}+\gamma_{5} s=0 \\
y_{2} y_{3} y_{4} y_{5} y_{6} y_{7}+y_{3} y_{4} y_{5} y_{6} y_{7} y_{8}+y_{6} y_{7} y_{8} y_{2} y_{3}+\gamma_{6} s=0 \\
y_{2} y_{3} y_{4} y_{5} y_{6} y_{7} y_{8}+y_{5} y_{6} y_{7} y_{8} y_{2} y_{3}+\gamma_{7} s=0 \\
y_{2} y_{3} y_{4} y_{5} y_{6} y_{7} y_{8}-1+\gamma_{8} s=0
\end{array}\right.
$$

The mixed volume for this system equals 25 and is exact. Of the 25 solutions, eight solutions have $s=0$ and are thus proper solutions. Two of the eight solutions are real. Denoting $I=\sqrt{-1}$, we select the solution

$$
y_{2}=-\frac{1}{2}+\frac{I}{2}, y_{3}=-I, y_{4}=-1, y_{5}=-1+I, y_{6}=I, y_{7}=\frac{1}{2}-\frac{I}{2}, y_{8}=1-I .
$$

Then we look at the first term of the series

$$
\left\{\begin{array}{lll}
y_{1}=t & & \\
y_{2}=-\frac{1}{2}+\frac{I}{2} & +z_{2} t \\
y_{3}=-I & +z_{3} t \\
y_{4}=-1 & +z_{4} t \\
y_{5}=-1+I & +z_{5} t \\
y_{6}=I & +z_{6} t \\
y_{7}=\frac{1}{2}-\frac{I}{2} & +z_{7} t \\
y_{8}=1-I & +z_{8} t
\end{array}\right.
$$

To decide whether the solution is isolated or not, we need to find values for the coefficient of the second term in the expansion. Substituting the series in the system in $\mathbf{f}(\mathbf{y})=\mathbf{0}$ and selecting the lowest order terms in $t$ leads to an overdetermined linear system in the $z_{k}$ variables. Solving with Maple yields

$$
z_{2}=-\frac{1}{2}, z_{3}=-1+I, z_{4}=0, z_{5}=-1, z_{6}=1-I, z_{7}=\frac{1}{2}, z_{8}=1 .
$$

Substituting the series in $\mathbf{f}(\mathbf{y})$ and we see that the result is $O\left(t^{2}\right)$. 


\section{2 cyclic 12-roots}

Extrapolating on the tropism for cyclic 4-roots, we considered $\mathbf{v}=(1,-1,1,-1,1,-1,1,-1$, $1,-1,1,-1)$. For this tropism the first term of the Puiseux series expansion

$$
\begin{array}{llrl}
x_{1} & =t & x_{2} & =t^{-1}\left(\frac{1}{2}-\frac{1}{2} i \sqrt{3}\right) \\
x_{3} & =-t & x_{4} & =t^{-1}\left(-\frac{1}{2}-\frac{1}{2} i \sqrt{3}\right) \\
x_{5} & =t\left(-\frac{1}{2}+\frac{1}{2} i \sqrt{3}\right) & x_{6} & =t^{-1}\left(\frac{1}{2}+\frac{1}{2} i \sqrt{3}\right) \\
x_{7} & =-t & x_{8} & =t^{-1}\left(-\frac{1}{2}+\frac{1}{2} i \sqrt{3}\right) \\
x_{9} & =t & x_{10} & =t^{-1}\left(\frac{1}{2}+\frac{1}{2} i \sqrt{3}\right) \\
x_{11} & =t\left(\frac{1}{2}-\frac{1}{2} i \sqrt{3}\right) & x_{12} & =t^{-1}\left(-\frac{1}{2}-\frac{1}{2} i \sqrt{3}\right)
\end{array}
$$

makes the system entirely and exactly equal to zero. Because of the symmetry, we have five other solution curves of this type. This is an exact certificate that shows cyclic 12-roots has a curve of degree two. Note that 0.866025403784439 is close enough to $\sqrt{3} / 2$ for us to recognize. The numerical determination of algebraic numbers in general is done via the integer relation detection algorithm of [5], see also [4].

The initial root that led to this certificate was one of the roots of an initial form system with mixed volume - after adding one slack variable to make the system square - equal to 49,816 . Note that this number is much less than the mixed volume of the original system: 500,352 .

\section{Conclusions and Future Directions}

In this paper concepts of numerical algebraic geometry were applied — witness sets and endgames - to determine the orientation of tropisms which could lead to certificates for proper algebraic curves.

We sketched how a polyhedral method could pick up all proper algebraic curves defined by a polynomial system. To extend this to solution sets of any dimension, say $k$, one would need to consider Puiseux series with $k$ free parameters and look for $k$ dimensional cones of tropisms. This generalization leads to the development of multiparameter polyhedral homotopies which may be of independent interest to numerical analysis.

\section{References}

[1] D. Adrovic and J. Verschelde. Tropical algebraic geometry in Maple, a preprocessing algorithm for finding common factors to multivariate polynomials with approximate coefficients. arXiv:0809.0298v1 [math.AG].

[2] M. Alonso, T. Mora, G. Niesi, and M. Raimondo. Local parametrization of space curves at singular points. In B. Falcidieno, I. Herman, and C. Pienovi, editors, Computer Graphics and Mathematics, pages 61-90. Springer-Verlag, 1992. 
[3] J. Backelin. Square multiples n give infinitely many cyclic n-roots. Technical report, Matematiska Institutioned, Stockholsm Universitet, 1989.

[4] D. Bailey. Integer relation detection. Computing in Science Ef Engineering, 2(1):24-28, 2000.

[5] D. Bailey and H.R.P. Ferguson. Numerical results on relations between fundamental constants using a new algorithm. Mathematics of Computation, 53(188):649-656, 1989.

[6] D.J. Bates, J.D. Hauenstein, A.J. Sommese, and C.W. Wampler. Software for numerical algebraic geometry: a paradigm and progress towards its implementation. In M.E. Stillman, N. Takayama, and J. Verschelde, editors, Software for Algebraic Geometry, volume 148 of The IMA Volumes in Mathematics and Its Applications, pages 1-14. Springer-Verlag, 2008.

[7] G.M. Bergman. The logarithmic limit-set of an algebraic variety. Transactions of the American Mathematical Society, 157:459-469, 1971.

[8] D.N. Bernshtein. The number of roots of a system of equations. Functional Anal. Appl., 9(3):183-185, 1975. Translated from Funktsional. Anal. i Prilozhen., 9(3):1-4,1975.

[9] G. Björck and R. Fröberg. Methods to "divide out" certain solutions from systems of algebraic equations, applied to find all cyclic 8-roots. In M. Gyllenberg and L.E. Persson, editors, Analysis, Algebra and Computers in Math. research, volume 564 of Lecture Notes in Mathematics, pages 57-70. Dekker, 1994.

[10] L. Blum, F. Cucker, M. Shub, and S. Smale. Complexity and Real Computation. SpringerVerlag, 1998.

[11] T. Bogart, A.N. Jensen, D. Speyer, B. Sturmfels, and R.R. Thomas. Computing tropical varieties. Journal of Symbolic Computation, 42(1):54-73, 2007.

[12] A.D. Bruno. Power Geometry in Algebraic and Differential Equations, volume 57 of NorthHolland Mathematical Library. Elsevier, 2000.

[13] Yu.D. Burago and V.A. Zalgaller. Geometric Inequalities, volume 285 of Grundlehren der mathematischen Wissenschaften. Springer-Verlag, 1988.

[14] J. Canny and J.M. Rojas. An optimal condition for determining the exact number of roots of a polynomial system. In S.M. Watt, editor, Proceedings of the 1991 International Symposium on Symbolic and Algebraic Computation (ISSAC 1991), pages 96-101. ACM, 1991.

[15] E. Cattani and A. Dickenstein. Counting solutions to binomial complete intersections. J. Complexity, 23(1):82-107, 2007.

[16] D. Cox. What is a toric variety? In R. Goldman and R. Krasauskas, editors, Topics in Algebraic Geometry and Geometric Modeling, volume 334 of Contemporary Mathematics, pages 203-223. AMS, 2003.

[17] D. Cox, J. Little, and D. O'Shea. Using Algebraic Geometry, volume 185 of Graduate Texts in Mathematics. Springer-Verlag, 1998. 
[18] Y. Dai, S. Kim, and M. Kojima. Computing all nonsingular solutions of cyclic-n polynomial using polyhedral homotopy continuation methods. J. Comput. Appl. Math., 152(1-2):83-97, 2003.

[19] T. de Jong and G. Pfister. Local Analytic Geometry. Basic Theory and Applications. Vieweg, 2000 .

[20] J.P. Dedieu and M. Shub. Newton's method for overdetermined systems of equations. Math. Comp., 69(231):1099-1115, 1999.

[21] I.Z. Emiris and J.F. Canny. Efficient incremental algorithms for the sparse resultant and the mixed volume. J. Symbolic Computation, 20(2):117-149, 1995.

[22] J.C. Faugère. A new efficient algorithm for computing Gröbner bases $\left(f_{4}\right)$. Journal of Pure and Applied Algebra, 139(1-3):61-88, 1999. Proceedings of MEGA'98, 22-27 June 1998, Saint-Malo, France.

[23] T. Gao and T.Y. Li. Mixed volume computation for semi-mixed systems. Discrete Comput. Geom., 29(2):257-277, 2003.

[24] T. Gao, T.Y. Li, and M. Wu. Algorithm 846: MixedVol: a software package for mixed-volume computation. ACM Trans. Math. Softw., 31(4):555-560, 2005.

[25] R.J. Gardner. Geometric Tomography. Cambridge University Press, second edition, 2006.

[26] I.M. Gel'fand, M.M. Kapranov, and A.V. Zelevinsky. Discriminants, Resultants and Multidimensional Determinants. Birkhäuser, 1994.

[27] M. Giusti and J. Heintz. La détermination de la dimension et des points isolées d'une variété algébrique peuvent s'effectuer en temps polynomial. In D. Eisenbud and L. Robbiano, editors, Computational Algebraic Geometry and Commutative Algebra, Cortona 1991, volume XXXIV of Symposia Mathematica, pages 216-256. Cambridge University Press, 1993.

[28] M. Giusti and J. Heintz. Kronecker's smart, little black boxes. In DeVore, R.A. and Iserles, A. and Süli, E., editor, Foundations of Computational Mathematics, volume 284 of London Mathematical Society Lecture Note Series, pages 69-104. Cambridge University Press, 2001.

[29] M. Giusti, J. Heintz, J.E. Morais, and L.M. Pardo. When polynomial equation systems can be "solved" fast? In G. Cohen, M. Giusti, and T. Mora, editors, Applied Algebra, Algebraic Algorithms and Error-Correcting Codes. 11th International Symposium, AAECC11. Paris, France, July 1995, volume 948 of Lecture Notes in Computer Science, pages 205-231. Springer-Verlag, 1995.

[30] M. Giusti, G. Lecerf, and B. Salvy. A gröbner free alternative for polynomial system solving. J. Complexity, 17(1):154-211, 2001.

[31] M. Grötschel, L. Lovász, and A. Schrijver. Geometric Algorithms and Combinatorial Optimization, volume 2 of Algorithms and Combinatorics. Springer-Verlag, 2nd edition, 1993. 
[32] T. Gunji, S. Kim, M. Kojima, A. Takeda, K. Fujisawa, and T. Mizutani. PHoM - a polyhedral homotopy continuation method for polynomial systems. Computing, 73(1):55-77, 2004.

[33] M. Hampton and R. Moeckel. Finiteness of relative equilibria of the four-body problem. Invent. Math., 163:289-312, 2006.

[34] B. Huber and B. Sturmfels. A polyhedral method for solving sparse polynomial systems. Math. Comp., 64(212):1541-1555, 1995.

[35] B. Huber and J. Verschelde. Polyhedral end games for polynomial continuation. Numerical Algorithms, 18(1):91-108, 1998.

[36] A.N. Jensen. Algorithmic Aspects of Gröbner Fans and Tropical Varieties. PhD thesis, Department of Mathematical Sciences, University of Aarhus, 2007.

[37] A.N. Jensen. Computing Gröbner fans and tropical varieties in Gfan. In M.E. Stillman, N. Takayama, and J. Verschelde, editors, Software for Algebraic Geometry, volume 148 of The IMA Volumes in Mathematics and Its Applications, pages 33-46. Springer-Verlag, 2008.

[38] A.N. Jensen, H. Markwig, and T. Markwig. An algorithm for lifting points in a tropical variety. Collectanea Mathematica, 59(2):129-165, 2008.

[39] G. Jeronimo, G. Matera, P. Solernó, and A. Waissbein. Deformation techniques for sparse systems. To appear in Found. Comput. Math.

[40] B. Ya. Kazarnovskii. Truncation of systems of polynomial equations, ideals and varieties. Izvestiya: Mathematics, 63(3):535-547, 1999.

[41] A.G. Khovanskiǐ. Newton polyhedra and the genus of complete intersections. Functional Anal. Appl., 12(1):38-46, 1978. Translated from Funktsional. Anal. i Prilozhen. 12(1),5161,1978 .

[42] A.G. Khovanskiǐ. Addendum 3. Algebra and mixed volumes. In Geometric Inequalities by Yu.D Burago and V.A. Zalgaller, pages 182-207. Springer-Verlag, 1988.

[43] A.G. Kushnirenko. Newton Polytopes and the Bézout Theorem. Functional Anal. Appl., 10(3):233-235, 1976. Translated from Funktsional. Anal. i Prilozhen. 10(3),82-83,1976.

[44] T.L. Lee, T.Y. Li, and C.H. Tsai. HOM4PS-2.0: a software package for solving polynomial systems by the polyhedral homotopy continuation method. Computing, 83(2-3):109-133, 2008.

[45] M. Lejeune-Jalabert, B. Teissier, and J.-J. Risler. Clôture intégrale des idéaux et équisingularité. arXiv:0803.2369v1 [math.CV] 16 Mar 2008.

[46] T.Y. Li. Numerical solution of polynomial systems by homotopy continuation methods. In F. Cucker, editor, Handbook of Numerical Analysis. Volume XI. Special Volume: Foundations of Computational Mathematics, pages 209-304. North-Holland, 2003.

[47] T.Y. Li and T.L. Lee. Mixed volume computation: a revisit. Available from http://www.math.msu.edu/ li. 
[48] T.Y. Li, T. Sauer, and J.A. Yorke. The cheater's homotopy: an efficient procedure for solving systems of polynomial equations. SIAM J. Numer. Anal., 26(5):1241-1251, 1989.

[49] J. Maurer. Puiseux expansion for space curves. Manuscripta Math., 32:91-100, 1980.

[50] J. McDonald. Fractional power series solutions for systems of equations. Discrete Comput. Geom., 27(4):501-529, 2002.

[51] G. Mikhalkin. Amoebas of algebraic varieties and tropical geometry. In S. Donaldson, Ya. Eliashberg, and M. Gromov, editors, Different Faces of Geometry, volume 3 of International Mathematical Series, pages 257-300. Springer-Verlag, 2004.

[52] T. Mizutani and A. Takeda. DEMiCs: a software package for computing the mixed volume via dynamic enumeration of all mixed cells. In M.E. Stillman, N. Takayama, and J. Verschelde, editors, Software for Algebraic Geometry, volume 148 of The IMA Volumes in Mathematics and Its Applications, pages 59-79. Springer-Verlag, 2008.

[53] T. Mizutani, A. Takeda, and M. Kojima. Dynamic enumeration of all mixed cells. Discrete Comput. Geom., 37(3):351-367, 2007.

[54] A. Morgan. Solving polynomial systems using continuation for engineering and scientific problems. Prentice-Hall, 1987.

[55] A.P. Morgan and A.J. Sommese. Coefficient-parameter polynomial continuation. Appl. Math. Comput., 29(2):123-160, 1989. Errata: Appl. Math. Comput. 51:207(1992).

[56] A.P. Morgan, A.J. Sommese, and C.W. Wampler. A power series method for computing singular solutions to nonlinear analytic systems. Numer. Math., 63:391-409, 1992.

[57] A. Poteaux. Computing monodromy groups defined by plane curves. In J. Verschelde and S.M. Watt, editors, SNC'07. Proceedings of the 2007 International Workshop on SymbolicNumeric Computation, pages 239-246. ACM, 2007.

[58] A. Poteaux and M. Rybowicz. Towards a symbolic-numeric method to compute Puiseux series: the modular part. arXiv:0803.3027v1 [cs.SC] 20 Mar 2008.

[59] A. Poteaux and M. Rybowicz. Good reduction of Puiseux series and complexity of the Newton-Puiseux algorithm over finite fields. In D. Jeffrey, editor, Proceedings of the 2008 International Symposium on Symbolic and Algebraic Computation (ISSAC 2008), pages 239246. ACM, 2008.

[60] V. Puiseux. Recherches sur les fonctions algébriques. J. de Math. Pures et Appl., 15:365-380, 1850 .

[61] J. Richter-Gebert, B. Sturmfels, and T. Theobald. First steps in tropical geometry. In G.L. Litvinov and V.P. Maslov, editors, Idempotent Mathematics and Mathematical Physics, volume 377 of Contemporary Mathematics, pages 289-317. AMS, 2005.

[62] J.M. Rojas. Toric intersection theory for affine root counting. Journal of Pure and Applied Algebra, 136(1):67-100, 1999. 
[63] J.M. Rojas. Why polyhedra matter in non-linear equation solving. In R. Goldman and R. Krasauskas, editors, Topics in Algebraic Geometry and Geometric Modeling, volume 334 of Contemporary Mathematics, pages 293-320. AMS, 2003.

[64] R. Schneider. Convex Bodies: The Brunn-Minkowski Theory, volume 44 of Encyclopedia of Mathematics and its Applications. Cambridge University Press, 1993.

[65] A.J. Sommese and J. Verschelde. Numerical homotopies to compute generic points on positive dimensional algebraic sets. J. Complexity, 16(3):572-602, 2000.

[66] A.J. Sommese, J. Verschelde, and C.W. Wampler. Numerical decomposition of the solution sets of polynomial systems into irreducible components. SIAM J. Numer. Anal., 38(6):20222046, 2001.

[67] A.J. Sommese, J. Verschelde, and C.W. Wampler. Numerical irreducible decomposition using PHCpack. In M. Joswig and N. Takayama, editors, Algebra, Geometry, and Software Systems, pages 109-130. Springer-Verlag, 2003.

[68] A.J. Sommese, J. Verschelde, and C.W. Wampler. Solving polynomial systems equation by equation. In Algorithms in Algebraic Geometry, volume 146 of The IMA Volumes in Mathematics and Its Applications, pages 133-152. Springer-Verlag, 2008.

[69] A.J. Sommese and C.W. Wampler. Numerical algebraic geometry. In J. Renegar, M. Shub, and S. Smale, editors, The Mathematics of Numerical Analysis, volume 32 of Lectures in Applied Mathematics, pages 749-763. AMS, 1996. Proceedings of the AMS-SIAM Summer Seminar in Applied Mathematics. Park City, Utah, July 17-August 11, 1995, Park City, Utah.

[70] A.J. Sommese and C.W. Wampler. The Numerical solution of systems of polynomials arising in engineering and science. World Scientific Press, Singapore, 2005.

[71] B. Sturmfels. Gröbner Bases and Convex Polytopes, volume 8 of University Lecture Series. AMS, 1996.

[72] B. Sturmfels. Polynomial equations and convex polytopes. Amer. Math. Monthly, 105(10):907-922, 1998.

[73] B. Sturmfels. Solving Systems of Polynomial Equations. Number 97 in CBMS Regional Conference Series in Mathematics. AMS, 2002.

[74] T. Theobald. Computing amoebas. Experimental Mathematics, 11(4):513-526, 2002.

[75] R.R. Thomas. Lectures in Geometric Combinatorics, volume 33 of Student Mathematical Library. AMS, 2006.

[76] J. Verschelde. Algorithm 795: PHCpack: A general-purpose solver for polynomial systems by homotopy continuation. ACM Trans. Math. Softw., 25(2):251-276, 1999. Software available at http://www.math.uic.edu/ jan. 
[77] J. Verschelde. Toric Newton method for polynomial homotopies. J. Symbolic Computation, 29(4-5):777-793, 2000.

[78] J. Verschelde and K. Gatermann. Symmetric Newton polytopes for solving sparse polynomial systems. Adv. Appl. Math., 16(1):95-127, 1995.

[79] J. Verschelde, K. Gatermann, and R. Cools. Mixed-volume computation by dynamic lifting applied to polynomial system solving. Discrete Comput. Geom., 16(1):69-112, 1996.

[80] J. Verschelde, P. Verlinden, and R. Cools. Homotopies exploiting Newton polytopes for solving sparse polynomial systems. SIAM J. Numer. Anal., 31(3):915-930, 1994.

[81] R.J. Walker. Algebraic Curves. Princeton University Press, 1950.

[82] G.M. Ziegler. Lectures on Polytopes, volume 152 of Graduate Texts in Mathematics. SpringerVerlag, New York, 1995. 